\title{
Retrospective comparison of cytological and histological bone marrow morphology in adult antiretroviral-naïve and antiretroviral experienced human immunodeficiency virus-infected patients with peripheral blood cytopaenias
}

\author{
Suraya Naidoo ${ }^{a}$ and Vincent Louis Naicker ${ }^{b *}$ \\ a Department of Haematology, University of KwaZulu-Natal, Durban, South Africa \\ ${ }^{b}$ National Health Laboratory Services, University of KwaZulu-Natal, Durban, South Africa \\ *Corresponding author, email: naidoosuraya@yahoo.com
}

\begin{abstract}
Background: A cohort of human immunodeficiency virus (HIV)-positive patients presenting to a quaternary hospital in KwaZuluNatal, South Africa over a period of one year was identified and morphological analyses of their bone marrow aspirates and trephine biopsies (BMAT) undertaken.

Aim: To compare and contrast the bone marrow morphological features of antiretroviral (ARV)-naïve and ARV-experienced HIV-positive population with cytopaenias; correlate the findings with CD4 counts; and, draw conclusions on whether ARV are associated with specific bone marrow (BM) findings.

Method: Aspirate and trephine biopsy examinations to assess dysplasia of cell lineages, architectural changes, granulomas or infiltrates were performed by light microscopy.

Results: 74 BMAT were examined, of which 24 were from the ARV-naïve cohort and 50 from ARV-treated cohort. Within granulomas, higher rates of marrow infiltration by acid-fast bacilli was present in the ARV-naïve cohort than the ARV-experienced cohort (6/10 $(60 \%)$ versus $9 / 26(35 \%)$, respectively). Higher rates of pure red cell aplasia (PRCA) were detected in the ARV-experienced than the ARV-naive cohort ( $14 \%$ versus $4 \%$, respectively). No difference was seen in morphological features and overall dysplasia rates (70\% and $71 \%$ of ARV-naïve and ARV-experienced cohort, respectively). At CD4 $>200$ cells/ul, higher rates of dysplasia were seen in the ARV-naïve cohort (3/3 (100\%) than in the ARV-experienced cohort (13/20 (65\%)).

Conclusion: Similar dysplasia rates and morphological features in both cohorts suggest ARVs do not cause myelodysplasia. BM tuberculosis is more frequently detected in granulomas of ARV-naïve than ARV-experienced samples. ARVs are implicated in causation of PRCA.
\end{abstract}

Keywords: antiretroviral therapy, bone marrow, dysplasia, HIV, morphology, pure red cell aplasia, tuberculosis

\section{Introduction}

There are 6.3 million people living with HIV in South Africa, with a prevalence rate of $19.1 \%$ among adults aged $15-49$ years. ${ }^{1}$ Antiretroviral (ARV) therapy was rolled out in March 2004; and, in 2011, there were an estimated 1.79 million people on ARV. ${ }^{2}$ In April 2013, South Africa saw the advent of fixed dose combination (FDC) antiretroviral therapy.

As cytopaenias are common in HIV-positive patients, ${ }^{3}$ the haematology laboratory at the quaternary hospital, Inkosi Albert Luthuli Central Hospital (IALCH), in KwaZulu-Natal is often consulted for cytopaenias in HIV-positive patients, who may or may not be on ARVs. Workup often entails a bone marrow aspirate and trephine biopsy assessment to ascertain the cause of cytopaenias, often suspected to be due to bone marrow (BM) infiltration by tuberculosis (TB), the most common HIV coinfection in South Africa. ${ }^{4}$ South Africa currently has the highest rate of new tuberculosis cases in the world. ${ }^{5}$

Other causes for cytopaenias may be the direct manifestation of HIV on the haemopoietic system (secondary myelodysplasia); bone marrow infiltration by opportunistic infections; haematological and non-haematological neoplasms; or, bone marrow suppression by various drugs. Drugs implicated in inducing cytopaenias in HIV-positive patients include prophylactic agents that are used to prevent opportunistic infections, such as Trimethoprim and Sulfamethoxazole (Bactrim) ${ }^{6}$ which has the well-known side effect of agranulocytosis/neutropenia; and, drugs used for treatment of opportunistic infections, such as anti-tuberculosis drugs and ARV agents, including the well-documented lamivudine and zidovudine in PRCA. ${ }^{-10}$

South Africa bears a wealth of knowledge in HIV research. However, there have been no published studies comparing the morphology of bone marrows of HIV-positive patients with peripheral blood cytopaenias who are on ARV and those who are not on ARV in South Africa; and, only one study comparing these cohorts outside South Africa. ${ }^{11}$

In another report, Choi et al. described remarkable haematological recoveries within 6 months to 3 years in HIVpositive patients with peripheral blood cytopaenias, who were initiated on highly active antiretroviral therapy ${ }^{12}$; however, the bone marrow morphology in these patients was not studied.

The aims of our study were to compare and contrast the bone marrow morphological features of ARV-naïve and ARVexperienced HIV-positive population with cytopaenias; to correlate the findings with CD4 counts; and, to draw conclusions on whether ARVs are associated with specific bone marrow findings. 


\section{Materials and methoc \\ Sample selection}

The stored BMAT of HIV infected individuals that were being investigated for cytopaenias $(n=120)$ received at Inkosi Albert Luthuli Central Hospital (IALCH) National Health Laboratory Services (NHLS) Haematology laboratory between 01 July 2012 and 30 June 2013 were retrieved from the laboratory archives.

Inclusion criteria comprised:

(1) adults (age $\geq 18$ years);

(2) presence of a cytopaenia (i.e. anaemia defined as haemoglobin $(\mathrm{Hb})$ level $<11 \mathrm{~g} / \mathrm{dl}$ in females or $<12 \mathrm{~g} / \mathrm{dl}$ in males, leukopenia defined as white cell count (WCC) $<4 \times 10^{9}$ cells/l, and thrombocytopaenia as platelet count $\left.(\mathrm{Plt})<150 \times 10^{9} \mathrm{cells} / \mathrm{l}\right)$; and,

(3) clinical information (i.e. gender, baseline CD4 count (if on ARV), CD4 count at time of sampling, ARV regimen and, if available, the duration on treatment).

This information was extracted from the bone marrow request forms and filled out by the clinician. Where insufficient, further clinical information was extracted from the archived records of telephonic consultations to the haematology laboratory, chart review at the base (referring) hospital and/or the laboratory computer information system.

Exclusion criteria included BMAT which were performed solely for staging of previously diagnosed lymphomas or as follow-up BMAT for such cases; and, aparticulate or failed BMAT in which critical clinical information required for the study could not be ascertained (i.e. CD4 count at baseline; CD4 count at time of $B M A T$; whether patient was on ARVs; and, the ARV regimen).

\section{Sample Cohorts}

The samples were then divided into two cohorts: ARV-naïve HIV BMAT and ARV-treated HIV BMAT.

\section{Method}

Bone marrow aspirates stained with May-Grünwald-Giemsa were examined by light microscopy. Cellularity, adequacy, maturation and evidence of dysplasia in the haemopoietic lineages were assessed.

Trephine biopsies stained with haematoxylin and eosin $(\mathrm{H}+\mathrm{E})$ were assessed for cellularity, architectural pattern, adequacy of the haemopoietic cell lines, dysplasia of megakaryocytes, presence of granulomatous inflammation or an infiltrate. Further immuno/histochemical stains (including Ziehl-Neelsen and Periodic-acid Schiff (PAS)) were performed if granulomas or an infiltrate was present.

Cellularity was assessed qualitatively and definitively graded as hypocellular, normocellular or hypercellular.

In accordance with the current World Health Organization (WHO) 2008 guidelines, ${ }^{13}$ a dysplastic feature was considered present if it was displayed on $>10 \%$ of the lineage. At least 30 megakaryocytes were examined per specimen (unless the length of the biopsy did not allow for this). Dysmegakaryopoiesis included presence of micromegakaryocytes, hypolobation/ monolobation, multinucleation or widely separated nuclei.
Dyserythropoiesis was present if the following nuclear and cytoplasmic abnormalities were identified: nuclear budding; internuclear bridging; karyorrhexis; multinuclearity; binucleate/ multinucleate forms; megaloblastic features including nuclear:cytoplasmic maturation asynchrony; and, the presence of cytoplasmic vacuolation.

Dysgranulopoiesis was defined as the presence of small or giant forms, nuclear hypolobation (pelgeroid neutrophils), irregular segmentation/hypersegmentation, hypogranularity and presence of ring forms. ${ }^{13}$

Based on the morphological findings, the cause of cytopaenias was determined to be due to:

(1) Inadequate haematopoiesis in the presence of hypocellularity or hypoplastic cell lines,

(2) Ineffective haematopoiesis in the presence of dysplasia

(3) Increased peripheral destruction/sequestration where adequate haematopoiesis is present,

(4) Bone marrow infiltration by neoplasia,

(5) Bone marrow infiltration by infection when microorganisms are identified, and/or

(6) Possible bone marrow infiltration as determined by the presence of granulomas without evidence of microorganisms.

Multifactorial causes for the cytopenias were sited if a combination of the above coexisted.

\section{Statistical analysis}

Statistics were analysed in SPSS version 21.

Pearson Chi-squared test and Fisher's exact test was used to test if morphological findings differ between the ARV-naïve and ARVexperienced group. A $p$-value $<0.05$ was considered statistically significant.

\section{Results}

\section{Study population}

There were 120 bone marrow biopsies investigated for cytopaenias in HIV-positive patients from 01 July 2012 to 30 June 2013.

Forty-six (46) samples were excluded from the study. Twentyfour (24) were inadequate for assessment (aparticulate aspirate and no haemopoietic tissue representation on biopsy specimen) and 22 had insufficient clinical information (as detailed above in inclusion criteria).

A total of 74 bone marrows were permitted into the study, stratified into 2 cohorts:

ARV-naïve $(n=24)$ and ARV-experienced $(n=50)$.

\section{ARV-naïve: Bone marrows of HIV positive} patients not on ARV $(\boldsymbol{n}=\mathbf{2 4})$

Twenty-four of 74 (32.4\%) bone marrows were from patients who had not yet started ARV therapy, with a 1:1 male to female ratio. 
Table 1: Comparative of CD4 counts in ARV-naïve and ARV-experienced cohort

\begin{tabular}{l|c|c|}
\hline CD4 count (cells/uL) & ARV-naïve (\%) $(n=24)$ & ARV-experienced (\%) $(n=50)$ \\
\hline$<200$ & $88(21)$ & $60(30)$ \\
\hline$<100$ & $50(12)$ & $21(42)$ \\
\hline$<50$ & $33(8)$ & $9(18)$ \\
\hline$<10$ & $17(4)$ & $5(10)$ \\
\hline
\end{tabular}

Table 2: Frequency of cytopaenias in ARV-naïve and ARV-experienced cohort

\begin{tabular}{|l|c|c|}
\hline Cytopaenia & ARV-naïve \% $(n=24)$ & ARV-experienced $\%(n=50)$ \\
\hline Isolated anaemia & $17(4)$ & $22(11)$ \\
\hline Isolated thrombocytopaenia & $8(2)$ & $8(4)$ \\
\hline Isolated leucopaenia & $4(1)$ & $0(0)$ \\
\hline Bicytopaenia & $41(10)$ & $32(16)$ \\
\hline Pancytopaenia & $29(7)$ & $38(19)$ \\
\hline
\end{tabular}

Table 3: Comparison of median values in ARV-naïve and ARV-experienced cohort

\begin{tabular}{|l|c|c|}
\hline Median & ARV-naïve $(n=24)$ & ARV-experienced $(n=50)$ \\
\hline Age & 32 & 32 \\
\hline M:F & $1: 1$ & $1: 2$ \\
\hline CD4 (cells/ul) & 90 & 126 \\
\hline Hb in anemia $(\mathrm{g} / \mathrm{dl})$ & $8.0(3.6-15.9)$ & $7.9(4.1-15.7)$ \\
\hline WCC in leucopenia $\left(\mathrm{X} 10^{9}\right.$ cells/l) & $4.65(0.66-14.76)$ & $3.77(0.85-12.48)$ \\
\hline Plts in thrombocytopenia $\left(\mathrm{X} 10^{9} \mathrm{cells} / \mathrm{l}\right)$ & $60(5-411)$ & $104(6-386)$ \\
\hline
\end{tabular}

The median age was 32 yrs (21-58 years). Three (3) patients were on TB treatment.

The median CD4 count was 90 cells/ul (range 1 - 559 cells/ul). Table 1 shows the distribution of CD4 counts.

Twenty-nine percent (29\%) of samples showed pancytopenia, $41 \%$ showed a bicytopaenia, and $29 \%$ showed an isolated cytopenia, in which anaemia was the most common isolated cytopenia (57\%).

Table 2 tabulates the prevalence of pancytopaenia, bicytopaenia and isolated cytopaenia in this cohort.

Table 3 provides the median cell counts in those with anaemia, leucopaenia and thrombocytopaenia.

\section{Cellularity}

Assessment of cellularity showed the majority (59\%) were normocellular, $29 \%$ were hypercellular and $12 \%$ were hypocellular.

\section{Dysplasia}

Eleven of 24 (46\%) samples showed megakaryocytic dysplasia. The most common dysplastic feature was monolobation/ hypolobation (9/11 i.e. $82 \%$ ) followed by micromegakaryocytes (7/11 i.e. 64\%).

Dyserythropoiesis was observed in 9/24 (38\%) cases. Nuclear abnormalities were the most common feature $(9 / 11$ i.e. $81 \%)$, with megaloblastic features in 5/9 (56\%).
Dysgranulopoiesis was present in $25 \%$ of cases, pelgerised forms were the most common form of dysplasia identified.

\section{Architecture}

Twelve of 24 (50\%) cases showed architectural distortion, assigned to granulomatous inflammation in 10 of those 12 cases.

\section{Ziehl-Neelsen, Periodic-acid Schiff and special histochemical stains}

Ziehl-Neelsen and Periodic-acid Schiff (PAS) histochemical stains were conducted on each of the cases showing granulomatous inflammation, which identified acid-fast bacilli in $60 \%(6 / 10)$ of cases, which were all PAS-negative.

\section{Cause of cytopenias}

The causes of the cytopaenias in this cohort are listed in Table 4.

Multifactorial causes for the cytopaenia(s) were present in $71 \%$ $(17 / 24)$ of cases.

The one case that showed possible bone marrow infiltration by neoplasia identified a large cell infiltrate, which, after immunohistochemical stains were performed, was suggestive of Hodgkin lymphoma or anaplastic large cell lymphoma (Anaplastic lymphoma kinase (ALK) negative).

Isolated anaemia was present in $4 / 24$ cases. One case $(1 / 4)$ showed that the anaemia was due to PRCA, 2/4 showed bone infiltration by infection (Tuberculosis) due to the presence of acid-fast bacilli, whilst $1 / 4$ appeared multifactorial (possible increased peripheral destruction/sequestration with ineffective production). 
Table 4: Comparative bone marrow morphology findings

\begin{tabular}{|c|c|c|}
\hline & Not on ARV (\%) & On ARV $(\%)(n=50)$ \\
\hline \multicolumn{3}{|l|}{ Cellularity } \\
\hline Normocellular & $59(14)$ & $68(34)$ \\
\hline Hypocellular & $12(3)$ & $20(10)$ \\
\hline Hypercellular & $29(7)$ & $12(6)$ \\
\hline \multicolumn{3}{|l|}{ Dysplasia } \\
\hline Erythroid & $38(9)$ & $22(11)$ \\
\hline Myeloid & $25(6)$ & $40(20)$ \\
\hline \multicolumn{3}{|l|}{ Architecture } \\
\hline Normal & $50(12)$ & $40(20)$ \\
\hline Distorted & $50(12)$ & $60(30)$ \\
\hline Presence of AFB in granulomas & $60(6)$ & $35(9)$ \\
\hline Presence of a malignant infiltrate & $4(1)$ & $4(2)$ \\
\hline \multicolumn{3}{|l|}{ Cause of cytopaenias } \\
\hline Inadequate haematopoiesis & $17(4)$ & $38(19)$ \\
\hline Ineffective production & $67(16)$ & $60(30)$ \\
\hline Increased peripheral destruction/sequestration & $54(13)$ & $42(21)$ \\
\hline Bone marrow infiltration by infection & $25(6)$ & $20(10)$ \\
\hline Bone marrow infiltration by neoplasia & $4(1)$ & $4(2)$ \\
\hline Possible bone marrow infiltration & $13(3)$ & $24(12)$ \\
\hline PRCA & $4(1)$ & $14(7)$ \\
\hline
\end{tabular}

Thrombocytopaenia was present in $75 \%$ of cases. An isolated thrombocytopaenia in only $8 \%$, and thrombocytopaenia in association with anaemia was present in $38 \%$ of cases.

The cause of the thrombocytopaenia both isolated or in combination with anaemia was due to the combination of ineffective production and increased peripheral destruction/ sequestration in all cases, with only one case showing possible bone marrow infiltration.

An isolated leucopenia was present in one case (4\%), which showed that lymphopaenia was most likely due to CD4 depletion, secondary to HIV infection.

Leucopaenia in association with an anaemia, thrombocytopaenia or pancytopaenia was present in $46 \%$ cases. Morphological assessment of the marrow showed the leucopaenia to be due to bone marrow infiltration in $37 \%$ of cases, whilst leucopaenia due to inadequate leucocyte production was in $27 \%$ of cases, and the leucopenia was due to either ineffective production or increased peripheral sequestration in $36 \%$ of those cases.

\section{ARV-treated: Bone marrows of HIV-positive patients on ARV $(n=50)$ \\ Fifty of $74(68 \%)$ marrows were from the HIV-positive patients on ARV cohort.}

This cohort comprised 32 females and 18 males, with a ratio of 1.8:1.

The median age was 32 years (range 18-60 years). Nine patients were on TB treatment.
The median CD4 count was 125 cells/ul (range 1-690). The CD4 cell count distribution is summarised in Table 1.

Table 2 tabulates the frequency of pancytopaenia, bicytopaenia and isolated cytopaenias in this cohort. Thirty-eight percent (38\%) had pancytopaenia; 32\% had bicytopenia; and, 30\% $(15 / 50)$ had unicytopenia (73\% of these had an isolated anaemia; $27 \%$ an isolated thrombocytopaenia; and, there were no cases of an isolated leucopaenia). Bicytopaenia of a thrombocytopaenia with anaemia were present in $53 \%$ of cases.

Duration on ARV treatment is summarised in Table 5.

\section{Cellularity}

Sixty-eight percent (68\%) were normocellular; $20 \%$ hypocellular; and, $12 \%$ of cases were hypercellular.

\section{Dysplasia}

Sixty-six percent (66\%) of cases showed megakaryocytic dysplasia.

The most common dysplastic feature was micromegakaryocytes in $34 \%$ and hypolobation in $22 \%$ of cases.

Dyserythropoiesis was present in $11 / 50$ (22\%) of cases with the most common findings being megaloblastic features in $7 / 11$ $(63 \%)$ and nuclear abnormalities in 4/11 (36\%).

Dysgranulopoiesis was seen in 20/50 (40\%) with nuclear abnormalities including pelgerised neutrophils in $75 \%$, giant 
Table 5: Duration on ARV and presence of dysplasia

\begin{tabular}{|l|c|c|}
\hline Duration & $\%(n=50)$ & Dysplasia (\%) \\
\hline$<6$ months & $44(22)$ & 82 \\
\hline$>6$ months & $34(17)$ & 52 \\
\hline Unknown & $22(11)$ & - \\
\hline
\end{tabular}

forms in 35\% and hypersegmented neutrophils in $20 \%$ of dysplastic cases.

\section{Architecture}

Trephine biopsy examination revealed architectural distortion in $30 / 50$ cases in which $26 / 30$ of cases showed granulomatous inflammation.

\section{Ziehl-Neelsen and PAS stains}

Ziehl-Neelsen and PAS stains were conducted in all 26 bone marrows with granulomatous inflammation. Acid-fast bacilli were present in 35\% (Figure 1), in which one appeared PASpositive (Figure 2).

\section{Cause of cytopaenias}

The cause of cytopaenias in this cohort was multifactorial in the majority of cases (72\%) and is summarised in Table 4.

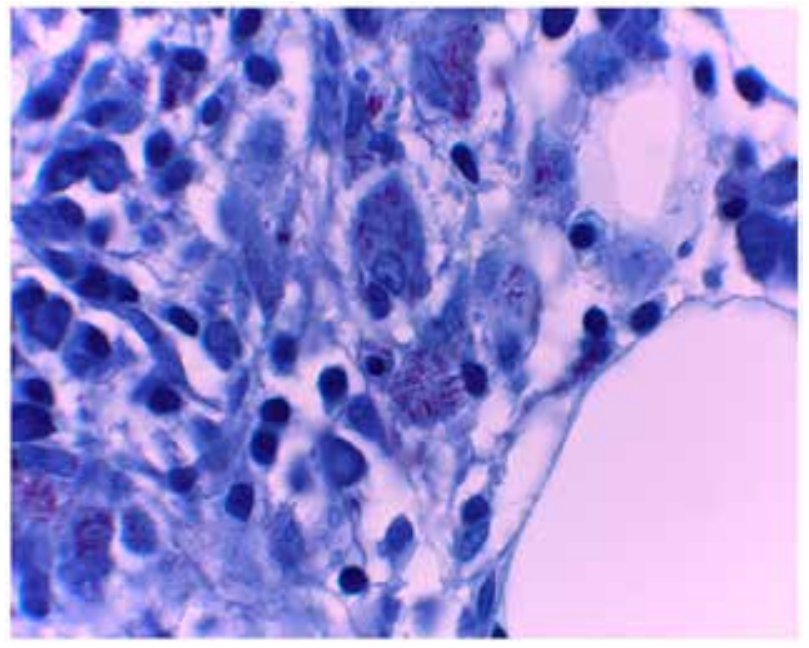

Note: Showing clumps of acid-fast bacilli.

Figure 1: Ziehl Neelsen 100× magnification.

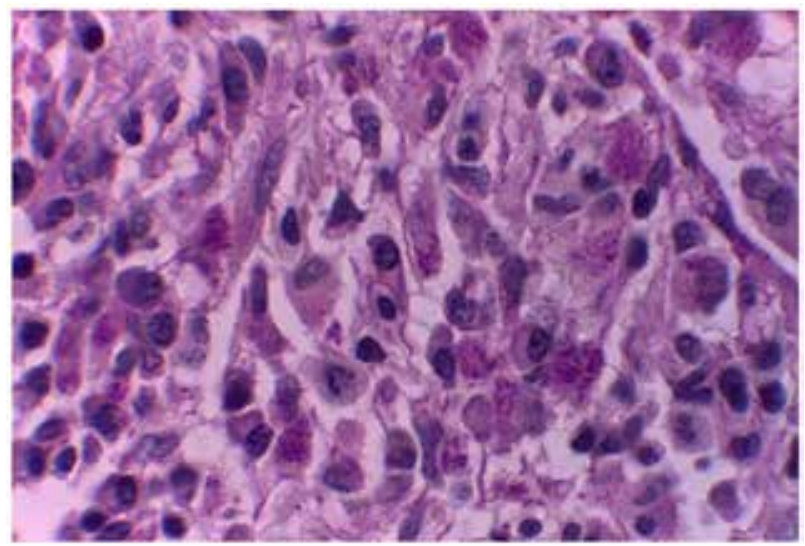

Of the 11 cases with an isolated anaemia, the cause was multifactorial in $55 \%$. Thirty-six (36\%) of these were due to PRCA Ineffective production was present in $45 \%$ of isolated anaemia cases, with $60 \%$ due to megaloblastic features, whilst $40 \%$ was most likely secondary to iron deficiency.

The cause of isolated thrombocytopaenia in the four cases was due to increased peripheral destruction/sequestration in all four cases with one case also showing evidence of ineffective production.

\section{Discussion}

To the best of our knowledge, there is only one other published report comparing bone marrow findings of ARV-experienced and ARV-naïve HIV-positive patients. ${ }^{11}$

Most other studies compared bone marrow morphology of AIDS and non-AIDS patients, irrespective of exposure to antiretrovirals. ${ }^{14-16}$

Limitations of this study included the small number of eligible samples. Eighteen (18\%) of the 120 samples received by the laboratory during the study period had to be excluded due to insufficient clinical information that was critical for study analysis. This impacted greatly on the sample size, limiting the value of inferential statistics.

As this was a retrospective study, another limitation was the inability to correlate findings with other investigations, such as microbiological studies (TB cultures of bone marrow aspirates) or virological results (e.g. Parvovirus B19 PCR in those with PRCA) that were not performed at the time of the BMAT sampling.

In our study, both cohorts showed similar findings with regards to cellularity, i.e. normocellular in most cases $(50-55 \%)$ correlating with findings in the study with such cohorts. ${ }^{11}$

Dysplasia in any lineage was present in $70 \%$ and $71 \%$ of the ARVnaïve and ARV-experienced cohort, respectively.

The megakaryocytic lineage was the most common lineage to show dysplasia in both cohorts. Although this finding was in stark contrast to most other studies of bone marrow morphology in HIV patients, which found no or limited dysplasia in the megakaryocytes ${ }^{11,14,17}$, Pande et al described similar rates and features of megakaryocytic dysplasia in their study of bone marrow changes in ARV-naïve anaemic patients. ${ }^{18}$ Nuclear cytoplasmic asynchrony suggestive of megaloblastic changes was a prominent dyserythropoietic finding, with other studies ${ }^{14,16,18}$ frequently reporting this finding. The most common feature of dysgranulopoiesis was abnormal nuclear segmentation (including pelgerised, ring, irregular forms and hypersegmentation) in both cohorts. This finding has been reported in other studies as a frequent granulocytic finding. ${ }^{14,16}$

Note: Showing PAS positive clumps of bacilli.

Figure 2: Periodic-acid Schiff (PAS) 100× magnification. 
Correlation of CD4 count and morphological features showed that at CD4 count $>200$ cells/ul (three in the ARV-naïve cohort and 20 in the ARV-experienced cohort), there was a higher rate of dysplasia in the ARV-naïve (3/3 i.e. $100 \%)$ than in the ARVexperienced cohort (13/20 i.e. $65 \%)$. Those on ARV therapy for less than 6 months showed a higher rate of dysplasia than those on ARV therapy for more than 6 months ( $82 \%$ versus $52 \%$ ). This is also summarised in Table 5.

Thus, the findings of similar rates and morphological features of dysplasia in both cohorts suggests that ARV drugs do not cause dysplasia. Moreover, ARV may improve marrow dysplasia thus the dysplasia seen in HIV is possibly reversible with ARV use. It may also be inferred that dysplasia improves after 6 months of ARV therapy. These observations were made on very small numbers, thus larger prospective studies paying specific attention to ARV regimens, duration of ARV use, monitoring of cytopenias and serial bone marrow morphological assessment will be invaluable in confirming these findings.

Granuloma formation was the reason for the architectural distortion in the majority of cases, specifically $50 \%$ and $60 \%$ in ARV-naïve and ARV-experienced cohorts, respectively.

The histochemical stains, Ziehl-Neelsen and Periodic-Acid Schiff (PAS) were conducted on all cases (i.e. 10 in ARV-naïve and 26 in ARV-treated cohorts) with granulomas. The presence of acid-fast bacilli within granulomas occurred in $60 \%$ and $35 \%$ of cases in the ARV-naïve and ARV-experienced cohorts, respectively.

TB of the bone marrow, as diagnosed by presence of PASnegative acid-fast bacilli within granulomas, was more common in the ARV-naïve cohort (60\%) than the ARV-experienced cohort (35\%), suggesting HIV-positive patients on ARV treatment are less likely to develop TB of the bone marrow.

Of the six cases in which acid-fast bacilli was identified in the ARV naïve cohort, all had CD4 counts of $<150$ cells/ul, with four having CD4 counts $\leq 20$ cells/ul.

In the ARV-experienced cohort, all except one patient had CD4 counts $<100$ cells/ul, in keeping with the fact that extrapulmonary TB occurs at advanced stages of HIV infection. ${ }^{19,20,21}$

PAS positively stained $1 / 36$ cases, confirming the diagnosis of Mycobacterium avium complex (MAC) in this one case.

This single diagnosed case of MAC was from the ARV-experienced cohort. The CD4 count at the time of the biopsy was 109 cells/ul, which was on an upward trend from the baseline CD4 count of 18 cells/ul 6 months prior to biopsy, a phenomenon seen in immune reconstitution inflammatory syndrome (IRIS). ${ }^{19,22}$

Bone marrow infiltration by a neoplastic process was diagnosed in only a minority of cases ( $4 \%$ in both cohorts), similar to a study conducted by van Schalkwyk et al. in South Africa in 2010 examining the utility of BMAT in patients with cytopenias and pyrexia of unknown origin. ${ }^{23}$

The diagnoses included possible Hodgkin Lymphoma in each cohort, similar to the findings in the above-mentioned study. ${ }^{23}$

All ARV-experienced patients with PRCA had CD4 counts $>100$ cell/ul.
PRCA was diagnosed in a total of eight patients, 7 from the ARVexperienced cohort, 2 of whom had a positive Parvovirus serology result, indicating past infection (IgG positive). However, no serology or Parvovirus polymerase chain reaction (PCR) results were available for all other patients.

The finding of PRCA in these patients suggests a possible role of ARV in the causation of PRCA. The ARV most commonly used in these cases was Lamivudine/3TC (71\%), Efavirenz/EFV (86\%), Zidovudine/AZT (29\%), Tenofovir (43\%) and Emtricitabine/FTC (29\%).

During the time period of the study, lamivudine was part of the initial ARV drug regimen for all HIV-positive patients in South Africa, whilst Efavirenz was one of two non-nucleoside reverse transcriptase inhibitors (NNRTI) used in the first line regimen.

In the latter part of the study period, fixed dose combination (FDC) ARVs were introduced. The initial FDC regimen included Tenofovir, Emtricitabine and Efavirenz, thus all used Emtricitabine as part of a FDC regimen.

Lamivudine and Zidovudine have been reported as drugs implicated in causation of PRCA. ${ }^{7-10}$ Efavirenz alone has never been reported as a cause of drug-induced PRCA. Although used in $86 \%(6 / 7)$ of the patients diagnosed with PRCA in this study, indicating a high probability as a causative agent, Efavirenz was used with one of the reported causative ARV drugs in PRCA (lamivudine or zidovudine) in $5 / 6$ patients. In the single patient who was not simultaneously exposed to lamivudine or zidovudine, there was no documented evidence of Parvovirus infection; and, it was not possible to exclude other causes of PRCA in this retrospective study.

Efavirenz use in this case was part of a FDC regimen. Tenofovir (which is used as a single agent or part of a FDC regimen) and Emtricitabine were also frequently used by the patients diagnosed with PRCA, suggesting possible causative effects. However, it is difficult to definitively establish a causal relationship between the individual ARV that is part of the fixed drug regimen and PRCA. Further studies are thus required on a larger cohort to establish possible causative ARVs (including the new fixed dose combination drugs) of PRCA.

All patients with PRCA in the ARV-experienced cohort had CD4 counts of $>100$ cells/ul, all showing an upward trend. This finding suggests not only that ARVs play a significant role in the development of PRCA but also that PRCA seems to occur during immunological reconstitution. Previous case reports also showed PRCA being diagnosed after starting ARV in HIV-positive patients with CD4 > 100 cells/ul, ${ }^{9}$ with diagnosis of IRIS-related Parvovirus B19 infection being considered. ${ }^{24}$ In our cases, Parvovirus B19 infection could not be definitively proven; however, in one case, parvovirus intranuclear inclusions were seen.

\section{Conclusion}

This retrospective analysis revealed many similarities and differences between the ARV-naïve and ARV-experienced cohort. A major drawback to our study was the limited number of samples that fulfilled the inclusion criteria, thus inferential statistics did not show any significant $p$-values.

None the less, similar morphological features of dysplasia in all cell lines were seen in both cohorts, suggesting that ARVs do not cause myelodysplasia. 
Higher rates of dysplasia in the ARV-naïve than in the ARVexperienced cohort at CD4 counts $>200$ cells/ul may imply HIVassociated myelodysplasia is reversible by improvement of CD4 count with ARV use.

Infiltration of the marrow by neoplasia was present in only $4 \%$ of samples in both cohorts, in which definitive diagnosis could not be made, revealing the limited value of bone marrow biopsies performed for diagnosis of malignancies in HIV-positive patients with cytopaenias.

The diagnosis of TB was more common in the ARV-naïve cohort suggesting that improved immune status, due to ARV use, prevents infection of the bone marrow by TB.

The diagnosis of PRCA was more common in the ARVexperienced cohort and in those who showed immunological response to highly active ARV therapy or combined ARV therapy. In this subgroup of ARV-experienced PRCA patients, the ARV most frequently used included Lamivudine, Zidovudine, Efavirenz, Tenofovir and Emtricitabine. Whilst Lamivudine and Zidovudine have been reported previously as possible causative agents in $\mathrm{PRCA}^{7-10}$, efavirenz, tenofovir and emtricitabine have never been reported. Further evaluation of these drugs and their role in PRCA is required. Due to study limitations, the role of Parvovirus B19 as the causation of PRCA could not be ascertained.

Acknowledgements - We would like to acknowledge Dr Julian Deonarian of Lancet Laboratories, Durban for his assistance with the figures, Dr Dashini Pillay for reviewing the manuscript and Dr T. K. Naidu for guidance during this study.

\section{References}

1. UNAIDS.2013. Available from:http://unaids.org/en/regionscountries/ countries/southafrica

2. Johnson LF. Access to antiretroviral treatment in South Africa 20042011. S Afr J HIV Med. 2012;13:22-7.

3. Moses A, Nelson J, Bagby GC Jr. The influence of human immunodeficiency virus-1 on hematopoiesis. Blood. 1998;91:147995.

4. World Health Organization. Global Tuberculosis report 2013. Geneva: WHO Press; 2013. p. 83.

5. World Health Organization. Global Tuberculosis report 2013. Geneva: WHO Press; 2013. p. 8-15.

6. Cruciani M. Antibacterial prophylaxis. Int J Antimicrob Agents. 2000;16:123-5.

7. Emmanuel N. Dessypris. Pure red cell aplasia. In: Hoffman R, Benz EJ, Shatill SJ, Furie B, Cohen HJ, Silberstein LE, McGlave P, editors.
Hematology basic principles and practice. 4th ed. Philadelphia, PA: Elsevier; 2005. p. 430-2.

8. John MA, Rhemtula YA, Menezes $C N$, et al. Lamivudine -induced red cell aplasia. J Med Microbiol. 2008;57:1032-5.

9. Balakrishnan $A$, Valsalan $R$, Sheshadri $S$, et al. Zidovudine-induced reversible pure red cell aplasia. Indian J Pharmacol. 2010;42:189-91.

10. Hassan A, Babadoko AA, Mamman A, et al. Zidovudine induced pure red cell aplasia: a case report. Nigerian J Med. 2009;18:332-3.

11. Dhurve SA, Dhurve AS. Bone marrow abonrmalities in HIV infection. Mediterr J Hematol Infect Dis. 2013;5(1):2013033.

12. Choi SY, Kim I, Kim NJ, et al. Hematological manifestations of Human Immunodeficiency virus infection and the effect of highly active antiretroviral therapy on cytopenia. Korean J Hematol. 2011;46:253-7.

13. Brunning RD, Orazi A, Germing U, et al. Myelodysplastic syndromes/ neoplasms, overview. In: Swerdlow SH, Campo E, Harris NL, et al., editors. WHO classification of tumours of the Haemopoietic and Lymphoid tissues. 4th ed. IARC: Lyon; 2008. p. 90-2.

14. Tripathi AK, Kalra P, Misra R, et al. Study of bone marrow abnormalities in patients with HIV disease. J Assoc Physicians India. 2005;53:105-10.

15. De Santis GC, Brunetta DM, Vilar FC, et al. Hematological abnormalities in HIV-infected patients. Int J Infect Dis. 2011;15:e808-11.

16. Mir N, Costello C, Luckit J, et al. HIV-disease and bone marrow changes: A study of 60 cases. Eur J Haematol. 1989;42:339-43.

17. Kaloutsi $V$, Kohlmeyer $U$, Maschek $H$, et al. Comparison of bone marrow and haematologic findings in patients with human immunodeficiency virus infection and those with myelodysplastic syndromes and infectious diseases. Am J Clin Pathol. 1994;101:123-9.

18. Pande A, Bhattacharyya M, Pain S, et al. Study of bone marrow changes in antiretroviral naive human immunodeficiency virusinfected anemic patients. Indian J Pathol Microbiol. 2011;54:542-6.

19. Riddell J, Kaul DR, Karakousis PC, et al. Mycobacterium avium complex immune reconstitution inflammatory syndrome: Long term outcomes. J Translational Med. 2007:5:50-6.

20. Hwang JH, Choe PG, Kim NH, et al. Incidence and risk factors of tuberculosis in patients with human immunodeficiency virus infection. J Korean Med Sci. 2013;28:374-7.

21. Jayral A, Raina R, Sarkar M, et al. Manifestations of tuberculosis in HIV/ AIDS patients and its relationship with CD4 count. Lung Ind: Official Org Ind Chest Soc. 2011;28:263-6.

22. Manosuthi W, Chaovavanich A, Tansuphaswadikul S, et al. Incidence and risk factors of major opportunistic infections after initiation of antiretroviral therapy among advanced HIV-infected patients in a resource-limited setting. J Infect. 2007;55:464-9.

23. Van Schalkwyk WA, Opie J, Novitzky N. The diagnostic utility of bone marrow biopsies performed for the investigation of fever and/or cytopenias in HIV-infected adults at Groote Schuur Hospital, Western Cape, South Africa. Int J Lab Hematol. 2011;33:258-66.

24. Watanabe $\mathrm{D}$, Taniguchi $\mathrm{T}$, Otani $\mathrm{N}$, et al. Immune reconstitution to parvovirus B19 and resolution of anemia in a patient treated with highly active antiretroviral therapy. J Infect Chemoth. 2011;17:283-7.

Received: 06-11-2014 Accepted: 21-08-2015 